Article

\title{
Luteal Support with very Low Daily Dose of Human Chorionic Gonadotropin after Fresh Embryo Transfer as an Alternative to Cycle Segmentation for High Responders Patients Undergoing Gonadotropin-Releasing Hormone Agonist-Triggered IVF
}

\author{
Andrea Roberto Carosso ${ }^{1, *}++^{(D)}$, Stefano Canosa ${ }^{1,+}\left(\mathbb{D}\right.$, Gianluca Gennarelli ${ }^{1}(\mathbb{D})$, Marta Sestero ${ }^{1}$,

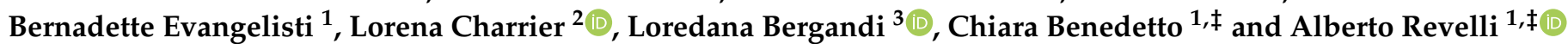 \\ 1 Obstetrics and Gynecology 1U, Physiopathology of Reproduction and IVF Unit, Department of Surgical \\ Sciences, Sant'Anna Hospital, University of Torino, 10042 Turin, Italy; s.canosa88@gmail.com (S.C.); \\ gennarellig1@gmail.com (G.G.); sesteromarta@gmail.com (M.S.); betty.evangelisti@gmail.com (B.E.); \\ chiara.benedetto@unito.it (C.B.); alberto.revelli@unito.it (A.R.) \\ 2 Department of Public Health and Pediatrics, University of Torino, Via Santena, 5 bis, 10126 Torino, Italy; \\ lorena.charrier@unito.it \\ 3 Department of Oncology, University of Torino, Via Santena 5 bis, 10126 Torino, Italy; \\ loredana.bergandi@unito.it \\ check for \\ updates \\ Citation: Carosso, A.R.; Canosa, S. \\ * Correspondence: andrea88.carosso@gmail.com; Tel.: +39-333-8111155 or +39-011-3135763 \\ + These authors contributed equally to this work. \\ $\ddagger$ These authors jointly supervised this work.
} Gennarelli, G.; Sestero, M.;

Evangelisti, B.; Charrier, L.; Bergandi, L.; Benedetto, C.; Revelli, A. Luteal Support with very Low Daily Dose of Human Chorionic Gonadotropin after Fresh Embryo Transfer as an

Alternative to Cycle Segmentation for High Responders Patients

Undergoing Gonadotropin-Releasing Hormone Agonist-Triggered IVF. Pharmaceuticals 2021, 14, 228. https://doi.org/10.3390/ph14030228

Academic Editor: Félix Carvalho

Received: 10 February 2021

Accepted: 3 March 2021

Published: 7 March 2021

Publisher's Note: MDPI stays neutral with regard to jurisdictional claims in published maps and institutional affiliations.

Copyright: (c) 2021 by the authors. Licensee MDPI, Basel, Switzerland. This article is an open access article distributed under the terms and conditions of the Creative Commons Attribution (CC BY) license (https:// creativecommons.org/licenses/by/ $4.0 /)$.
Abstract: The segmentation of the in vitro fertilization (IVF) cycle, consisting of the freezing of all embryos and the postponement of embryo transfer (ET), has become popular in recent years, with the main purpose of preventing ovarian hyperstimulation syndrome (OHSS) in patients with high response to controlled ovarian stimulation (COS). Indeed cycle segmentation (CS), especially when coupled to a GnRH-agonist trigger, was shown to reduce the incidence of OHSS in high-risk patients. However, CS increases the economic costs and the work amount for IVF laboratories. An alternative strategy is to perform a fresh ET in association with intensive luteal phase pharmacological support, able to overcome the negative effects of the GnRH-agonist trigger on the luteal phase and on endometrial receptivity. In order to compare these two strategies, we performed a retrospective, reallife cohort study including 240 non-polycystic ovarian syndrome (PCO) women with expected high responsiveness to $\mathrm{COS}$ (AMH $>2.5 \mathrm{ng} / \mathrm{mL}$ ), who received either fresh ET plus 100 IU daily human chorionic gonadotropin (hCG) as luteal support (FRESH group, $n=133$ ), or cycle segmentation with freezing of all embryos and postponed ET (CS group, $n=107)$. The primary outcomes were: implantation rate (IR), live birth rate (LBR) after the first ET, and incidence of OHSS. Overall, significantly higher IR and LBR were observed in the CS group than in the FRESH group (42.9\% vs. $27.8 \%, p<0.05$ and $32.7 \%$ vs. $19.5 \%, p<0.05$, respectively); the superiority of CS strategy was particularly evident when 16-19 oocytes were retrieved (LBR $42.2 \%$ vs. 9.5\%, $p=0.01$ ). Mild OHSS appeared with the same incidence in the two groups, whereas moderate and severe OHSS forms were observed only in the FRESH group ( $1.5 \%$ and $0.8 \%$, respectively). In conclusion, in non-PCO women, high responders submitted to COS with the GnRH-antagonist protocol and GnRH-agonist trigger, CS strategy was associated with higher IR and LBR than the strategy including fresh ET followed by luteal phase support with a low daily hCG dose. CS appears to be advisable, especially when $>15$ oocytes are retrieved.

Keywords: luteal phase support; corpus luteum function; GnRH agonist trigger; hCG; GnRH antagonist protocol; IVF; ovarian hyperstimulation syndrome 


\section{Introduction}

In reproductive physiology, the luteinizing hormone (LH) is mandatory for maintaining corpus luteum function and promotes the secretion of progesterone and growth factors involved in embryo implantation and placentation [1,2].

In in vitro fertilization (IVF), suppression of hypothalamic activity by GnRH analogs/ antagonists is required during controlled ovarian stimulation (COS) of multiple follicles in order to avoid a premature LH surge. Final oocyte maturation is commonly triggered by administering exogenous human chorionic gonadotropin (hCG). Indeed, due to its strong biological activity and its relatively long half-life [3], hCG is able to sustain the multiple corpora lutea throughout the luteal phase. Nevertheless, in order to compensate for the supra-physiological pre-ovulatory levels of estradiol, observed during COS [4,5], the addition of progesterone is needed for optimal endometrial receptivity and to avoid early pregnancy loss $[6,7]$.

In women who have a large ovarian reserve (defined as "high responders"), the administration of exogenous hCG in the presence of supraphysiological estradiol levels is associated with an increased risk of ovarian hyperstimulation syndrome (OHSS) [8], partly caused by the stimulating effects of hCG on ovarian production of vasoactive factors $[9,10]$.

The risk of OHSS may be minimized using "short protocols" with GnRH antagonists to accomplish COS, and triggering ovulation with a bolus of GnRH-agonist instead of hCG [11-13]. With a GnRH-agonist trigger, a physiological peak of LH is responsible for final oocyte maturation. Whereas the number of retrieved mature oocytes is usually comparable after either a GnRH-agonist or hCG trigger [11], the rapid drop of endogenous LH concentration observed after a GnRH-agonist trigger negatively affects corpus luteum function, in turn worsening endometrial receptivity [14]. Indeed, luteal phase deficiency after GnRH-agonist trigger administration is extremely frequent, resulting in lower implantation and clinical pregnancy rates [15], even when progesterone supplementation is used.

In order to solve this problem, freezing of all embryos and delaying embryo transfer to the following ovarian cycle has been proposed. This strategy is defined as cycle segmentation (CS) or "freeze-all" strategy. With CS, the risk of OHSS is strongly reduced, whereas IVF efficacy is maintained [16]. However, such an approach increases both the economic costs and the amount of work for the IVF unit. Furthermore, some patients consider the delay of embryo transfer (ET) negatively, and their anxiety level increases, enhancing the risk of drop-out.

As alternatives to CS, some forms of intensive pharmacological supplementation of the luteal phase after the GnRH-agonist trigger have been suggested. Among others, the addition of estradiol to progesterone at high doses [17,18], or the administration of 1500 IU hCG the day of oocyte pick up (OPU) [14,19]. Promising results have been obtained with the daily administration of a very low (100 IU) dose of hCG in the luteal phase, which was proposed in a small series of patients who underwent $\mathrm{GnRH}$-agonist trigger and fresh ET [20].

The aim of the present study was to compare the outcome of this approach, that is, fresh ET followed by a very low daily dose of hCG, with CS, applied to a group of non-PCO patients treated with a GnRH-analogue trigger, who were expected to be high responders on the basis of their ovarian reserve markers. The primary end-points were the implantation rate (IR), the live birth rate (LBR), and the ovarian hyperstimulation syndrome (OHSS) incidence.

\section{Results}

The baseline clinical characteristics of the women included in the study are shown in Table 1. Table 2 shows the outcome of IVF in the two study groups. 
Table 1. Clinical baseline characteristics of the patients receiving fresh embryo transfer (ET) plus human chorionic gonadotropin (hCG) luteal supplementation (FRESH group) vs. those undergoing cycle segmentation with freezing of all embryos and delayed ET (CS group). Data are expressed as mean \pm standard deviation.

\begin{tabular}{cccc}
\hline Clinical Baseline Characteristics & $\begin{array}{c}\text { FRESH } \\
(\boldsymbol{n}=\mathbf{1 3 3 )}\end{array}$ & $\begin{array}{c}\text { CS } \\
(\boldsymbol{n}=\mathbf{1 0 7})\end{array}$ & $\boldsymbol{p}$-Value \\
\hline Age (years) & $34.7 \pm 4.1$ & $34.8 \pm 4.1$ & 0.80 \\
BMI (kg/cm $\left.{ }^{2}\right)$ & $22.6 \pm 3.7$ & $22.8 \pm 4.6$ & 0.79 \\
Basal AMH (ng/mL) & $5.3 \pm 2.9$ & $7.4 \pm 5.6$ & $<0.01$ \\
Basal (day 3) FSH (UI/L) & $6.4 \pm 1.4$ & $6.4 \pm 1.7$ & 0.91 \\
Basal (day 3) LH (UI/L) & $6.6 \pm 3.1$ & $7.3 \pm 3.5$ & 0.14 \\
Antral follicle count (AFC) & $19.9 \pm 6.8$ & $26.9 \pm 10.1$ & $<0.01$ \\
\hline
\end{tabular}

LH: luteinizing hormone.

Table 2. In vitro fertilization (IVF) outcomes of patients receiving fresh ET plus hCG luteal supplementation (FRESH group) vs. those undergoing cycle segmentation with freezing of all embryos and delayed ET (CS group). Data are expressed as mean \pm standard deviation and median (IQR), if appropriate, for quantitative variables and percentages for qualitative variables. * Endometrial thickness in the FRESH group was measured during oocyte pick up (OPU), whereas in the CS group, it was measured the day after LH urinary peak during the spontaneous cycle in which ET with thawed embryo was performed. ${ }^{* *}$ In both groups, only the first ET was considered. OHSS: ovarian hyperstimulation syndrome.

\begin{tabular}{|c|c|c|c|}
\hline IVF Cycle Outcomes & $\begin{array}{c}\text { FRESH } \\
(n=133)\end{array}$ & $\begin{array}{c}\text { CS } \\
(n=107)\end{array}$ & $p$-Value \\
\hline Mean daily FSH dose (UI) & $169.4 \pm 38.9$ & $164.8 \pm 40.1$ & \multirow{2}{*}{0.40} \\
\hline Median (IQR) & $150(150-194)$ & $150(150-187.5)$ & \\
\hline Total FSH dose (UI) & $1774 \pm 557.8$ & $1659.9 \pm 519.4$ & \multirow{2}{*}{0.21} \\
\hline Median (IQR) & $1650(1350-2000)$ & $1600(1350-1948)$ & \\
\hline Estradiol at ovulation trigger & $2332.1 \pm 1026.2$ & $3573.8 \pm 2163.2$ & \multirow[b]{2}{*}{$<0.01$} \\
\hline $\begin{array}{c}(\mathrm{pg} / \mathrm{mL}) \\
\text { Median (IQR) }\end{array}$ & $2142(1554-2966)$ & $3203(2265-4363)$ & \\
\hline Endometrial thickness $(\mathrm{mm})$ * & $10.3 \pm 1.7$ & $10 \pm 2$ & 0.14 \\
\hline Retrieved oocytes $(n)$ & $12.6 \pm 3.1$ & $14.2 \pm 3.4$ & \multirow{2}{*}{$<0.01$} \\
\hline Median (IQR) & $13(10-14)$ & $15(11-17)$ & \\
\hline Mature (MII) oocytes $(n)$ & $10.3 \pm 3.1$ & $11 \pm 3.6$ & 0.11 \\
\hline Fertilization rate $(\%)$ & $63.7 \pm 19.9$ & $70.8 \pm 20.9$ & \multirow{2}{*}{$<0.01$} \\
\hline Median (IQR) & $66.7(50-77.8)$ & $72.2(55.5-90)$ & \\
\hline Mean embryo score $(n)$ & $7 \pm 1.4$ & $7.2 \pm 1.5$ & \multirow{2}{*}{0.55} \\
\hline Median (IQR) & $7.1(6.1-7.9)$ & $7.1(6.4-8.2)$ & \\
\hline Blastocysts $(n)$ & $3.3 \pm 2.6$ & $4.5 \pm 2.7$ & \multirow{2}{*}{$<0.01$} \\
\hline Median (IQR) & $3(1-5)$ & $4(2-6)$ & \\
\hline Implantation rate $(\%) * *$ & 27.8 & 42.9 & $<0.05$ \\
\hline Clinical pregnancy rate/first ET\% ** & 26.3 & 40.2 & $<0.05$ \\
\hline Live birth rate/first ET\% ** & 19.5 & 32.7 & $<0.05$ \\
\hline Mild OHSS\% & 12 & 12.1 & 0.98 \\
\hline Moderate OHSS\% & 1.5 & 0 & - \\
\hline Severe OHSS\% & 0.8 & 0 & - \\
\hline
\end{tabular}

Women in the CS group had higher ovarian reserve markers (AMH and AFC): although the mean and total dose of exogenous FSH was comparable in the two groups, they finally got a higher number of retrieved oocytes and blastocysts (Table 2). However, the mean embryo score was comparable in the two groups. The higher responsiveness to COS in the CS group did not influence the calculation of the implantation rate (IR), clinical pregnancy rate (CPR), or the live birth rate (LBR), as it was performed taking into account only the first ET, and did not cumulatively consider all ETs. As per the protocol, all viable 
and transferable blastocysts in the CS group were vitrified, but those after the first ET were not considered to calculate the outcomes.

Overall, we observed significantly higher IR, CPR and LBR after the first ET in the CS group than in the FRESH group (IR $=42.9 \%$ vs. $27.8 \%, p<0.05$; CPR $=40.2 \%$ vs. $26.3 \%$, $p<0.05 ;$ LBR $=32.7 \%$ vs. $19.5 \%, p<0.05$, respectively).

Due to the significant association between the number of retrieved oocytes and the study groups $(p<0.01)$, patients were then stratified according to the number of retrieved oocytes (Table 3; $\mathrm{A}=8-11$ oocytes, $\mathrm{B}=12-15$ oocytes, $\mathrm{C}=16-19$ oocytes) to compare the FRESH and CS groups in three subsets of patients with almost no significant differences in the baseline characteristics and with comparable responsiveness to COS. Even after normalizing for the response to COS, which virtually abolished all effects due to different ovarian reserve markers in the two "naïve" groups, we observed that the CS strategy led to higher, although not statistically significant, percentage of positive hCG test and LBR, and that its superiority was particularly remarkable when $>15$ oocytes were retrieved (Table 3). These results were confirmed after fitting logistic regression models in the three subgroups of women, where LBR was the dependent variable, and the study group (CS vs. FRESH), $\mathrm{AMH}$ and estradiol levels were the independent variables: after adjusting for $\mathrm{AMH}$ and estradiol levels, the CS group showed a higher probability of LBR in all subgroups, with the association being nearly significant (OR 4.43, CI95\% 0.84; 23.2, $p=0.07$, data not shown) among women with $>15$ retrieved oocytes.

Table 3. Clinical baseline characteristics and IVF outcome of the patients receiving fresh ET plus hCG luteal supplementation (FRESH group) vs. those undergoing cycle segmentation with freezing of all embryos and delayed ET (CS group). Women were stratified according to the number of retrieved oocytes in order to normalize for the ovarian response to COS: $\mathrm{A}=8-11$ retrieved oocytes; $\mathrm{B}=12-15$ retrieved oocytes; $\mathrm{C}=16-19$ retrieved oocytes. Data are expressed as mean \pm standard deviation or median (IQR), as appropriate, if not otherwise stated. ${ }^{* *}$ In both groups, only the first ET was considered.

\begin{tabular}{|c|c|c|c|}
\hline A (8-11 Retrieved Oocytes) & $\begin{array}{l}\text { FRESH } \\
(n=52)\end{array}$ & $\begin{array}{c}\text { CS } \\
(n=30)\end{array}$ & $p$-Value \\
\hline Age (years) & $34.8 \pm 4.3$ & $34.9 \pm 4.2$ & 0.94 \\
\hline BMI $\left(\mathrm{kg} / \mathrm{cm}^{2}\right)$ & $23.1 \pm 3.4$ & $23.6 \pm 6.2$ & 0.63 \\
\hline Basal AMH (ng/mL) & $4.15(2.8-6)$ & $5.2(3.3-6.5)$ & 0.13 \\
\hline $\begin{array}{l}\text { Estradiol at ovulation trigger } \\
\qquad(\mathrm{pg} / \mathrm{mL})\end{array}$ & $2070(1529-2920.5)$ & $2485.5(1746-4268)$ & 0.06 \\
\hline Positive hCG test $\%(n)$ & $25(13)$ & $30(9)$ & 0.62 \\
\hline Live birth rate/first ET $\%(n) * *$ & $21.2(11)$ & $23.3(7)$ & 1.00 \\
\hline B (12-15 Retrieved Oocytes) & $\begin{array}{l}\text { FRESH } \\
(n=60)\end{array}$ & $\begin{array}{c}\text { CS } \\
(n=32)\end{array}$ & \\
\hline Age (years) & $34.7 \pm 4.1$ & $34.4 \pm 3.7$ & 0.73 \\
\hline $\mathrm{BMI}\left(\mathrm{kg} / \mathrm{cm}^{2}\right)$ & $22.1 \pm 3.3$ & $22.5 \pm 3.4$ & 0.63 \\
\hline Basal AMH (ng/mL) & $5.45(3.4-6.3)$ & $6.3(3.8-8.7)$ & 0.07 \\
\hline $\begin{array}{l}\text { Estradiol at ovulation trigger } \\
\qquad(\mathrm{pg} / \mathrm{mL})\end{array}$ & $2284.5(1609.5-3090)$ & $3254.5(2342-4311)$ & $<0.01$ \\
\hline Positive hCG test $\%(n)$ & $31.7(19)$ & $40.6(13)$ & 0.49 \\
\hline Live birth rate/first ET \% $(n)^{* *}$ & $21.7(13)$ & $28.1(9)$ & 0.61 \\
\hline C (16-19 Retrieved Oocytes) & $\begin{array}{c}\text { Fresh } \\
(n=21)\end{array}$ & $\begin{array}{c}\text { CS } \\
(n=45)\end{array}$ & \\
\hline Age (years) & $34.3 \pm 3.8$ & $35.1 \pm 4.3$ & 0.48 \\
\hline $\mathrm{BMI}\left(\mathrm{kg} / \mathrm{cm}^{2}\right)$ & $22.8 \pm 5.5$ & $22.4 \pm 4.1$ & 0.76 \\
\hline Basal AMH (ng/mL) & $5.7(4.3-6.5)$ & $6.9(4.4-9.8)$ & 0.07 \\
\hline $\begin{array}{l}\text { Estradiol at ovulation trigger } \\
\qquad(\mathrm{pg} / \mathrm{mL})\end{array}$ & 1918 (1583-3024) & 3345 (2445-4363) & $<0.01$ \\
\hline Positive hCG test $\%(n)$ & $33.3(7)$ & $51.1(23)$ & 0.19 \\
\hline Live birth rate/first $\mathrm{ET} \%(n) * *$ & $9.5(2)$ & $42.2(19)$ & 0.01 \\
\hline
\end{tabular}


A total number of 29 events of mild OHSS were observed, with no significant difference between the two groups (12\% vs. 12.1\%). Moderate and severe OHSS were observed with two and one cases in the FRESH group, respectively (1.5\% and $0.8 \%$, respectively, Table 3$)$, whereas no cases were reported in the CS group (Table 2).

\section{Discussion}

In recent years, the freezing of all available embryos followed by thaw ET in one of the subsequent spontaneous cycles, namely known as "cycle segmentation", has become quite popular. The main reason for this is the efficacy in preventing OHSS in high-risk patients, among which are both women with PCO ovaries and those with very marked responsiveness to COS, the so-called "high responders" [21-25]. These patients are usually submitted to a COS protocol, including the use of low-dose gonadotropins plus a GnRH-antagonist to prevent premature LH peak. The trigger of final follicular maturation is obtained using a single bolus of GnRH-agonist, which stimulates the release of an endogenous LH peak.

In comparison with the classical hCG trigger, the GnRH-agonist-stimulated LH peak remarkably lowers the risk of inducing OHSS, but it is shorter and biologically weaker, resulting in insufficient support for the corpus luteum during the luteal phase [26]. This does not represent an issue when all embryos are vitrified and ET is postponed to one of the following cycles.

Besides the documented efficacy in preventing OHSS, CS may offer further advantages, such as a more favorable pattern of endometrial gene expression and increased receptivity [26], both linked to the absence of detrimental endometrial effects caused by excessive E2 levels and/or premature P elevation [27]. Indeed, COS has been associated with altered angiogenesis, impaired placentation [28-30], and higher instability of the reproductive tract microbiota [31], all factors able to reduce the likelihood of blastocyst implantation. As a matter of fact, in specific subgroups of patients, CS have been reported to be associated with higher implantation rates [21,32] and better perinatal and obstetric outcomes $[33,34]$ than the conventional fresh ET strategy. It is debated whether the application of CS might be extended even to cases not necessarily at risk of OHSS.

However, the weak points of CS mean it is not always convenient; it is undoubtedly more time-consuming and economically expensive for the IVF laboratory, and it may even represent an additional emotional burden for the patients, who may live with increasing anxiety the delay of their ET.

For these reasons, even in patients at risk of OHSS, alternative strategies allowing fresh ET after the use of GnRH-analogue trigger have been conceived [6]. In fact, there is evidence that even very low levels of hCG might adequately support the luteal phase in GnRHagonist triggered cycles, even without the addition of exogenous progesterone [20,35].

Over the last few years, the strategy of performing fresh ET and supplement the luteal phase with very low doses of hCG has been used in our IVF unit, as an alternative to traditional CS, in the case of non-PCO patients with ovarian reserve markers and a wide ovarian reserve $(\mathrm{AMH}>2.5 \mathrm{ng} / \mathrm{mL}, \mathrm{AFC}>15)$, who were expected to deliver a high response to COS without the very high OHSS risk typical of patients with PCOS. When these patients obtained less than 20 oocytes at OPU, and the ultrasound (US) endometrial appearance at OPU was satisfactory (thickness $>7 \mathrm{~mm}$ and type 1 echogenicity), a fresh ET was scheduled, and the luteal phase was supplemented using $100 \mathrm{IU} / \mathrm{d}$ s.c. hCG. In contrast, when $>19$ oocytes were retrieved and/or the endometrium appeared thinner than $7 \mathrm{~mm}$, or with type 2 or 3 US appearance, all blastocysts were vitrified, and ET was postponed in a subsequent, natural cycle with suitable endometrial characteristics.

The main finding of the present retrospective investigation is that the CS strategy allowed the obtaining of higher IR, CPR, and LBR, considering only the first ET than the strategy including fresh ET and hCG supplementation. This suggests that a very low daily dose of hCG, although overall safe with respect to the risk of OHSS, is probably inadequate to compensate for the negative effect of the trigger GnRH-agonist bolus on the luteal function. 
Indeed, women in the CS group had significantly higher AMH and AFC and, as expected, a better ovarian responsiveness to COS, more oocytes retrieved, and more available blastocysts. It is well known that there is a strong relationship between the number of oocytes and the LBR in a fresh IVF cycle, with optimal outcomes with between 15-20 eggs [36]. However, even normalizing for ovarian responsiveness through stratification in three subgroups of patients with a comparable number of retrieved oocytes and similar basal "a priori" characteristics, the analyses showed a better performance of the CS strategy, especially evident among patients retrieving $>15$ oocytes.

Notably, in the FRESH group, the LBR/ET was lower in the subgroup with 16-19 retrieved oocytes than in the two subgroups with fewer oocytes $(p<0.01)$, suggesting that when peak estradiol levels are higher, the inadequacy of hCG supplementation in restoring endometrial receptivity is probably more marked [37].

Conversely, the LBR/ET in the CS group showed an opposite trend, steadily increasing with the number of retrieved oocytes, an observation that confirms the association between ovarian responsiveness and the likelihood of getting a live birth [36].

As for safety issues, the strategy of performing a fresh ET with very low dose hCG supplementation was associated with an overall rate of OHSS (2.2\% moderate plus severe OHSS rate), which compares favorably with the incidence of 3-6\% commonly reported overall for IVF cycles [38]. However, it is questionable whether this rate of OHSS could still be acceptable, as the elimination of OHSS represents one of the main current goals of IVF treatments.

\section{Materials and Methods}

The study was designed as a retrospective, real-life cohort study and was performed at the IVF Unit of Sant'Anna University Hospital after obtaining the approval of the Institutional Review Board, in accordance with the Helsinki Committee requirements (n. 0040486, 23/04/2020).

Among 2312 women undergoing IVF in the years 2016-2019, 434 were classified as expected high responders, according to their ovarian reserve markers (AMH $>2.5 \mathrm{ng} / \mathrm{mL}$ and antral follicle count (AFC) > 15). Of those women, 298 had regular menstrual cycles, no signs of hyperandrogenism, and no PCO appearance of the ovaries at transvaginal US; they were included in the study as non-PCO expected high responders.

The remaining 136 women had polycystic ovary syndrome (PCO) according to ESHRE guidelines [39] and were excluded from the study. PCO patients, in fact, are known to represent a specific population that is at very high risk of developing severe OHSS, and according to our protocols, CS is mandatory.

Other 58 women with multifollicular non-PCO ovaries were excluded from the study after OPU because $>19$ oocytes were retrieved, and, again, our protocols forced us to perform CS due to the well-known, direct relationship between the number of retrieved oocytes and the risk of severe OHSS.

Therefore, finally, we compared 133 non-PCO high responders receiving fresh ET plus luteal phase hCG supplementation (FRESH group) vs. 107 comparable patients receiving cycle segmentation (CS group).

COS, OPU, and IVF or ICSI were performed as described in Appendix A. Both groups received a single subcutaneous injection of $0.2 \mathrm{mg}$ Triptorelin Acetate (Decapetyl, Ferring, Germany) as an ovulation trigger. Embryos were assessed morphologically on day 2 using the 1-10 point scale score by Holte et al. [40], and then again on day 5 according to The Istanbul Consensus Workshop [41]. Embryos that progressed to the expanded blastocyst stage on day 5 (score 3) showed compacted inner cell mass (score 1), and cohesive trophoectoderm (score 1 or 2) were selected for the first transfer in both groups. The ET was performed as previously described [42].

The decision on whether patients would receive fresh ET or CS was taken during OPU and was based on the endometrial US appearance; when the endometrial thickness was $<7 \mathrm{~mm}$ and/or its appearance was not trilinear (so it was type 2 or 3), CS was performed. In 
contrast, when the endometrial thickness was $\geq 7 \mathrm{~mm}$, with a trilinear (type 1) appearance, fresh ET was chosen.

In the FRESH group, women received a single blastocyst fresh ET on day 5. Starting the day after OPU, 100 IU/day of hCG (Gonasi HP, Ibsa, Lugano, Switzerland) were administered subcutaneously for 14 days.

In the CS group, a standard vitrification protocol was used to freeze all blastocysts. ET was performed in one of the following menstrual cycles, monitoring spontaneous ovulation by urinary LH-detection kit (Clearblue ${ }^{\circledR}$ Ovulation Test, Swiss Precision Diagnostics, Switzerland). ET with one thawed blastocyst was performed only whether endometrial thickness reached at least $7 \mathrm{~mm}$, with an endometrial US type 1 appearance the day after urinary LH peak. The luteal phase was supplemented with $180 \mathrm{mg}$ /day intra-vaginal natural progesterone (Crinone 8, Merck, Germany), starting $48 \mathrm{~h}$ after the LH peak and continuing for 14 days.

In case of a positive pregnancy test, transvaginal ultrasound (TV-US) examination was scheduled two weeks later.

The implantation rate (IR) was calculated, taking into account also the biochemical pregnancies. The clinical pregnancy rate (CPR) was calculated considering only pregnancies that were confirmed at TV-US (presence of a gestational sac) two weeks after the positive pregnancy test. Live birth rate (LBR) was calculated with live birth defined as a live-born infant after $>24$ weeks of gestation.

The number of retrieved oocytes is a well-known factor affecting the LBR [36]; to minimize the impact of this variable, a sub-analysis was performed, in which FRESH and CS groups were compared after stratification for the number of retrieved oocytes into three subgroups (8-11, 12-15, or 16-19).

Safety was evaluated considering the incidence of OHSS, whose severity was assessed as previously described [43]. Statistical comparison between the two study groups was performed using the GraphPad Prism V7 software package and Stata16; the Student's $t$-test or the non-parametric Wilcoxon rank-sum test were used, as appropriate, for continuous variables (shown as mean $\pm \mathrm{SD}$ and/or median and interquartile range (IQR)), whereas the Chi squared or Fisher's tests were used for categorical variables (shown as percentages and absolute frequencies). Multivariable analysis models were then used to investigate the independent effects of the study group (CS vs. FRESH) on LBR in the three subgroups of women, stratified according to the number of retrieved oocytes. Logistic regression models were fit using LBR as dependent variable and study group, with AMH and estradiol levels as independent variables. All statistical tests were two-sided, and a $p$-value of 0.05 was considered the significance threshold.

\section{Conclusions}

In conclusion, to the best of our knowledge, this is the largest study comparing IVF outcomes in non-PCO high responders submitted to COS with GnRH-antagonist protocol, GnRH-agonist trigger, and either CS or fresh ET with hCG luteal phase support.

With the limitation of its retrospective nature, the present study shows that the strategy of CS is associated with higher IR, CPR, and LBR than the alternative strategy, including fresh ET plus repeated very low daily dose of hCG.

If confirmed using a larger sample size and in a randomized controlled trial, the present findings would help to orient clinical decisions, with the final aim of improving IVF outcomes.

Author Contributions: A.R.C. and M.S. conceived the study and wrote the manuscript. S.C. performed data analysis and co-wrote the manuscript. A.R. and L.B. critically revised the manuscript. S.C., M.S., and B.E. collected the data. G.G. contributed to data analysis. L.C. performed the statistical analysis. C.B. coordinated the study. All authors reviewed and approved the final version of the manuscript.

Funding: This research received no external funding. 
Institutional Review Board Statement: All procedures performed in studies involving human participants were in accordance with the ethical standards of the institutional and/or national research committee and with the 1964 Helsinki Declaration and its later amendments or comparable ethical standards. The study was approved by the Bioethics Committee of the Medical University of Torino (n. 0040486, 23/04/2020).

Informed Consent Statement: Informed consent was obtained in accordance with the rules of the Bioethics Committee of the Medical University of Torino.

Data Availability Statement: All data are available under request to the authors

Acknowledgments: The authors wish to thank all the staff of the IVF Unit of S. Anna hospital.

Conflicts of Interest: The authors have no conflicts of interest to declare.

\section{Appendix A}

All the enrolled non-PCO expected high responders underwent COS with the GnRHantagonist regimen, using recombinant FSH (Gonal F, Merck, Darmstadt, Germany or Puregon, MSD, Readington, NJ, USA). The FSH starting dose ranged from 100 to 150 IU according to age and BMI and was then adjusted from stimulation day 5-7 according to the ovarian response at the first checkpoint, during which transvaginal US (TV-US) and serum estradiol (E2) measurement were performed. From stimulation day 5, a daily injection ( $0.25 \mathrm{mg} / \mathrm{d}$ s.c) of GnRH-antagonist (Cetrorelix Merck, Darmstadt, Germany or Orgalutran, MSD, Readington, NJ, USA) was added. Follicular growth was monitored by TV-US and serum E2 every 2-3 days, and when at least two follicles reached $18 \mathrm{~mm}$ mean diameter, with appropriate E2 circulating levels, ovulation was triggered by a single subcutaneous injection of $0.2 \mathrm{mg}$ Triptorelin Acetate (Decapetyl, Ferring, Saint-Prex, Switzerland). Ovulation trigger was performed in the same way in the FRESH and CS groups. OPU was performed approximately $36 \mathrm{~h}$ after Triptorelin Acetate injection by US-guided aspiration under local anesthesia (paracervical block). Follicular fluid was immediately observed under a stereomicroscope; cumulus-oocyte complexes (COCs) were washed in buffered medium (Flushing medium, Cook Ltd., Limerick, Ireland), and oocytes were inseminated within $4 \mathrm{~h}$ from OPU using either conventional IVF or ICSI, according to the quality of the semen sample. Normal fertilization was confirmed when the presence of two pronuclei $(2 \mathrm{PN})$ and the extrusion of the second polar body were observed 16-18 h after oocyte insemination. Zygotes were placed in 4-well dishes (Thermo Ficher Scientific, Waltham, MA, USA) and cultured in pre-equilibrated cleavage medium (Cook Ltd., Limerick, Ireland) overlain with mineral oil (Culture Oil, Cook Ltd., Limerick, Ireland).

\section{References}

1. Devoto, L.; Fuentes, A.; Kohen, P.; Céspedes, P.; Palomino, A.; Pommer, R.; Muñoz, A.; Strauss, J.F. The Human Corpus Luteum: Life Cycle and Function in Natural Cycles. Fertil. Steril. 2009, 92, 1067-1079. [CrossRef]

2. Tesarik, J.; Hazout, A.; Mendoza, C. Luteinizing Hormone Affects Uterine Receptivity Independently of Ovarian Function. Reprod. Biomed. Online 2003, 7, 59-64. [CrossRef]

3. Damewood, M.D.; Shen, W.; Zacur, H.A.; Schlaff, W.D.; Rock, J.A.; Wallach, E.E. Disappearance of Exogenously Administered Human Chorionic Gonadotropin. Fertil. Steril. 1989, 52, 398-400. [CrossRef]

4. Tavaniotou, A.; Devroey, P. Effect of Human Chorionic Gonadotropin on Luteal Luteinizing Hormone Concentrations in Natural Cycles. Fertil. Steril. 2003, 80, 654-655. [CrossRef]

5. Fauser, B.C.J.M.; Devroey, P. Reproductive Biology and IVF: Ovarian Stimulation and Luteal Phase Consequences. Trends Endocrinol. Metab. 2003, 14, 236-242. [CrossRef]

6. Humaidan, P.; Papanikolaou, E.G.; Kyrou, D.; Alsbjerg, B.; Polyzos, N.P.; Devroey, P.; Fatemi, H.M. The Luteal Phase after GnRH-Agonist Triggering of Ovulation: Present and Future Perspectives. Reprod. Biomed. Online 2012, 24, 134-141. [CrossRef]

7. Van der Linden, M.; Buckingham, K.; Farquhar, C.; Kremer, J.A.M.; Metwally, M. Luteal Phase Support for Assisted Reproduction Cycles. Cochrane Database Syst. Rev. 2015, CD009154. [CrossRef] [PubMed]

8. La Marca, A.; Sighinolfi, G.; Radi, D.; Argento, C.; Baraldi, E.; Artenisio, A.C.; Stabile, G.; Volpe, A. Anti-Mullerian Hormone $(\mathrm{AMH})$ as a Predictive Marker in Assisted Reproductive Technology (ART). Hum. Reprod. Update 2010, 16, 113-130. [CrossRef] [PubMed] 
9. Neulen, J.; Yan, Z.; Raczek, S.; Weindel, K.; Keck, C.; Weich, H.A.; Marmé, D.; Breckwoldt, M. Human Chorionic GonadotropinDependent Expression of Vascular Endothelial Growth Factor/Vascular Permeability Factor in Human Granulosa Cells: Importance in Ovarian Hyperstimulation Syndrome. J. Clin. Endocrinol. Metab. 1995, 80, 1967-1971. [CrossRef]

10. Albert, C.; Garrido, N.; Mercader, A.; Rao, C.V.; Remohí, J.; Simón, C.; Pellicer, A. The Role of Endothelial Cells in the Pathogenesis of Ovarian Hyperstimulation Syndrome. Mol. Hum. Reprod. 2002, 8, 409-418. [CrossRef]

11. Humaidan, P.; Kol, S.; Papanikolaou, E.G. Copenhagen GnRH Agonist Triggering Workshop Group GnRH Agonist for Triggering of Final Oocyte Maturation: Time for a Change of Practice? Hum. Reprod. Update 2011, 17, 510-524. [CrossRef]

12. Orvieto, R. Can We Eliminate Severe Ovarian Hyperstimulation Syndrome? Hum. Reprod. 2005, 20, 320-322. [CrossRef]

13. Griesinger, G.; von Otte, S.; Schroer, A.; Ludwig, A.K.; Diedrich, K.; Al-Hasani, S.; Schultze-Mosgau, A. Elective Cryopreservation of All Pronuclear Oocytes after GnRH Agonist Triggering of Final Oocyte Maturation in Patients at Risk of Developing OHSS: A Prospective, Observational Proof-of-Concept Study. Hum. Reprod. 2007, 22, 1348-1352. [CrossRef]

14. Iliodromiti, S.; Blockeel, C.; Tremellen, K.P.; Fleming, R.; Tournaye, H.; Humaidan, P.; Nelson, S.M. Consistent High Clinical Pregnancy Rates and Low Ovarian Hyperstimulation Syndrome Rates in High-Risk Patients after GnRH Agonist Triggering and Modified Luteal Support: A Retrospective Multicentre Study. Hum. Reprod. 2013, 28, 2529-2536. [CrossRef] [PubMed]

15. Humaidan, P.; Bredkjaer, H.E.; Bungum, L.; Bungum, M.; Grøndahl, M.L.; Westergaard, L.; Andersen, C.Y. GnRH Agonist (Buserelin) or HCG for Ovulation Induction in GnRH Antagonist IVF/ICSI Cycles: A Prospective Randomized Study. Hum. Reprod. 2005, 20, 1213-1220. [CrossRef]

16. Devroey, P.; Polyzos, N.P.; Blockeel, C. An OHSS-Free Clinic by Segmentation of IVF Treatment. Hum. Reprod. 2011, 26, 2593-2597. [CrossRef]

17. Lukaszuk, K.; Liss, J.; Lukaszuk, M.; Maj, B. Optimization of Estradiol Supplementation during the Luteal Phase Improves the Pregnancy Rate in Women Undergoing in Vitro Fertilization-Embryo Transfer Cycles. Fertil. Steril. 2005, 83, 1372-1376. [CrossRef]

18. Ismail Madkour, W.A.; Noah, B.; Abdel Hamid, A.M.S.; Zaheer, H.; Al-Bahr, A.; Shaeer, M.; Moawad, A. Luteal Phase Support with Estradiol and Progesterone versus Progesterone Alone in GnRH Antagonist ICSI Cycles: A Randomized Controlled Study. Hum. Fertil. (Camb) 2016, 19, 142-149. [CrossRef] [PubMed]

19. Santos-Ribeiro, S.; Mackens, S.; Popovic-Todorovic, B.; Racca, A.; Polyzos, N.P.; Van Landuyt, L.; Drakopoulos, P.; de Vos, M.; Tournaye, H.; Blockeel, C. The Freeze-All Strategy versus Agonist Triggering with Low-Dose HCG for Luteal Phase Support in IVF/ICSI for High Responders: A Randomized Controlled Trial. Hum. Reprod. 2020. [CrossRef]

20. Andersen, C.Y.; Fischer, R.; Giorgione, V.; Kelsey, T.W. Micro-Dose HCG as Luteal Phase Support without Exogenous Progesterone Administration: Mathematical Modelling of the HCG Concentration in Circulation and Initial Clinical Experience. J. Assist. Reprod. Genet. 2016, 33, 1311-1318. [CrossRef]

21. Roque, M. Freeze-All Policy: Is It Time for That? J. Assist. Reprod. Genet. 2015, 32, 171-176. [CrossRef]

22. Davenport, M.J.; Vollenhoven, B.; Talmor, A.J. Gonadotropin-Releasing Hormone-Agonist Triggering and a Freeze-All Approach: The Final Step in Eliminating Ovarian Hyperstimulation Syndrome? Obstet. Gynecol. Surv. 2017, 72, 296-308. [CrossRef] [PubMed]

23. Acharya, K.S.; Acharya, C.R.; Bishop, K.; Harris, B.; Raburn, D.; Muasher, S.J. Freezing of All Embryos in in Vitro Fertilization Is Beneficial in High Responders, but Not Intermediate and Low Responders: An Analysis of 82,935 Cycles from the Society for Assisted Reproductive Technology Registry. Fertil. Steril. 2018, 110, 880-887. [CrossRef] [PubMed]

24. Revelli, A.; Carosso, A.; Grassi, G.; Gennarelli, G.; Canosa, S.; Benedetto, C. Empty Follicle Syndrome Revisited: Definition, Incidence, Aetiology, Early Diagnosis and Treatment. Reprod. Biomed. Online 2017, 35, 132-138. [CrossRef]

25. Evans, J.; Hannan, N.J.; Edgell, T.A.; Vollenhoven, B.J.; Lutjen, P.J.; Osianlis, T.; Salamonsen, L.A.; Rombauts, L.J.F. Fresh versus Frozen Embryo Transfer: Backing Clinical Decisions with Scientific and Clinical Evidence. Hum. Reprod. Update 2014, 20 , 808-821. [CrossRef]

26. Gonen, Y.; Balakier, H.; Powell, W.; Casper, R.F. Use of Gonadotropin-Releasing Hormone Agonist to Trigger Follicular Maturation for in Vitro Fertilization. J. Clin. Endocrinol. Metab. 1990, 71, 918-922. [CrossRef]

27. Venetis, C.A.; Kolibianakis, E.M.; Bosdou, J.K.; Tarlatzis, B.C. Progesterone Elevation and Probability of Pregnancy after IVF: A Systematic Review and Meta-Analysis of over 60000 Cycles. Hum. Reprod. Update 2013, 19, 433-457. [CrossRef]

28. Haouzi, D.; Assou, S.; Mahmoud, K.; Tondeur, S.; Rème, T.; Hedon, B.; De Vos, J.; Hamamah, S. Gene Expression Profile of Human Endometrial Receptivity: Comparison between Natural and Stimulated Cycles for the Same Patients. Hum. Reprod. 2009, 24, 1436-1445. [CrossRef]

29. Maheshwari, A.; Bhattacharya, S. Elective Frozen Replacement Cycles for All: Ready for Prime Time? Hum. Reprod. 2013, 28, 6-9. [CrossRef]

30. Amor, D.J.; Xu, J.X.; Halliday, J.L.; Francis, I.; Healy, D.L.; Breheny, S.; Baker, H.W.G.; Jaques, A.M. Pregnancies Conceived Using Assisted Reproductive Technologies (ART) Have Low Levels of Pregnancy-Associated Plasma Protein-A (PAPP-A) Leading to a High Rate of False-Positive Results in First Trimester Screening for Down Syndrome. Hum. Reprod. 2009, 24, 1330-1338. [CrossRef]

31. Carosso, A.; Revelli, A.; Gennarelli, G.; Canosa, S.; Cosma, S.; Borella, F.; Tancredi, A.; Paschero, C.; Boatti, L.; Zanotto, E.; et al. Controlled Ovarian Stimulation and Progesterone Supplementation Affect Vaginal and Endometrial Microbiota in IVF Cycles: A Pilot Study. J. Assist. Reprod. Genet. 2020. [CrossRef] 
32. Roque, M.; Valle, M.; Guimarães, F.; Sampaio, M.; Geber, S. Freeze-All Cycle for All Normal Responders? J. Assist. Reprod. Genet. 2017, 34, 179-185. [CrossRef]

33. Kansal Kalra, S.; Ratcliffe, S.J.; Milman, L.; Gracia, C.R.; Coutifaris, C.; Barnhart, K.T. Perinatal Morbidity after in Vitro Fertilization Is Lower with Frozen Embryo Transfer. Fertil. Steril. 2011, 95, 548-553. [CrossRef]

34. Sha, T.; Yin, X.; Cheng, W.; Massey, I.Y. Pregnancy-Related Complications and Perinatal Outcomes Resulting from Transfer of Cryopreserved versus Fresh Embryos in Vitro Fertilization: A Meta-Analysis. Fertil. Steril. 2018, 109, 330-342.e9. [CrossRef] [PubMed]

35. Andersen, C.Y.; Elbaek, H.O.; Alsbjerg, B.; Laursen, R.J.; Povlsen, B.B.; Thomsen, L.; Humaidan, P. Daily Low-Dose HCG Stimulation during the Luteal Phase Combined with GnRHa Triggered IVF Cycles without Exogenous Progesterone: A Proof of Concept Trial. Hum. Reprod. 2015, 30, 2387-2395. [CrossRef]

36. Sunkara, S.K.; Rittenberg, V.; Raine-Fenning, N.; Bhattacharya, S.; Zamora, J.; Coomarasamy, A. Association between the Number of Eggs and Live Birth in IVF Treatment: An Analysis of 400135 Treatment Cycles. Hum. Reprod. 2011, 26, 1768-1774. [CrossRef]

37. Joo, B.S.; Park, S.H.; An, B.M.; Kim, K.S.; Moon, S.E.; Moon, H.S. Serum Estradiol Levels during Controlled Ovarian Hyperstimulation Influence the Pregnancy Outcome of in Vitro Fertilization in a Concentration-Dependent Manner. Fertil. Steril. 2010, 93, 442-446. [CrossRef]

38. Schirmer, D.A.; Kulkarni, A.D.; Zhang, Y.; Kawwass, J.F.; Boulet, S.L.; Kissin, D.M. Ovarian Hyperstimulation Syndrome after Assisted Reproductive Technologies: Trends, Predictors, and Pregnancy Outcomes. Fertil. Steril. 2020, 114, 567-578. [CrossRef]

39. Costello, M.F.; Misso, M.L.; Balen, A.; Boyle, J.; Devoto, L.; Garad, R.M.; Hart, R.; Johnson, L.; Jordan, C.; Legro, R.S.; et al. Evidence Summaries and Recommendations from the International Evidence-Based Guideline for the Assessment and Management of Polycystic Ovary Syndrome: Assessment and Treatment of Infertility. Hum. Reprod. Open 2019, 2019, hoy021. [CrossRef]

40. Holte, J.; Berglund, L.; Milton, K.; Garello, C.; Gennarelli, G.; Revelli, A.; Bergh, T. Construction of an Evidence-Based Integrated Morphology Cleavage Embryo Score for Implantation Potential of Embryos Scored and Transferred on Day 2 after Oocyte Retrieval. Hum. Reprod. 2007, 22, 548-557. [CrossRef]

41. Alpha Scientists in Reproductive Medicine and ESHRE Special Interest Group of Embryology The Istanbul Consensus Workshop on Embryo Assessment: Proceedings of an Expert Meeting. Hum. Reprod. 2011, 26, 1270-1283. [CrossRef]

42. Revelli, A.; Gennarelli, G.; Biasoni, V.; Chiadò, A.; Carosso, A.; Evangelista, F.; Paschero, C.; Filippini, C.; Benedetto, C. The Ovarian Sensitivity Index (OSI) Significantly Correlates with Ovarian Reserve Biomarkers, Is More Predictive of Clinical Pregnancy than the Total Number of Oocytes, and Is Consistent in Consecutive IVF Cycles. J. Clin. Med. 2020, 9, 1914. [CrossRef] [PubMed]

43. Ovarian Hyperstimulation Syndrome, Management (Green-Top Guideline No. 5). Available online: https:/ /www.rcog.org.uk/ en/guidelines-research-services / guidelines/gtg5/ (accessed on 30 June 2020). 